DOI: $10.2478 / \mathrm{v} 10122-010-0002-1$

\title{
L'ASSIMILATION VOCALIQUE RÉGRESSIVE EN BAMANANKAN: DE DIFFÉRENTS CAS ${ }^{1}$
}

\author{
MOHAMED LARABI DiALLO
}

\begin{abstract}
Mohamed Larabi Diallo. L'assimilation vocalique régressive en bamanankan: de différents cas. Lingua Posnaniensis, vol. LII (1)/2010. Poznan Society for the Advancement of the Arts and Sciences. PL ISSN 0079-4740, ISBN 978-83-7654-030-6, pp. 15-38

The aim of this article is to furnish more information about the vocalic regressive assimilation, which is the most frequent phonological phenomenon in Bamanankan.

Nearby the adjectives, post-positions, adverbs, the plural morpheme and the distributive morpheme is described also the behaviour of some other conjonctions, particles, and particular cases of assimilation which have been not taken into account in the preceding article.

For the comments and remarks on some categories of word we will refer to the chapters concerning them. The description will always concern only the case where the two consecutive words present different vowels.

The language which is the topic of this contribution is called bámánánkán. The speakers are named bámánán. It is very known under the name bambara. The bamanankan is the most important language among the 13 languages which are recognized officially as national languages of the Republic of Mali. It belongs to the Manding which is a group of Mande Languages which are in their turn an under family of Niger-Congo.
\end{abstract}

Mohamed Larabi Diallo, DER Allemand, Faculté des Lettres, Langues, Arts et Sciences Humaines (FLASH), BP. E 3637, Université de Bamako, République du Mali

Dans le présent exposé nous tenterons de fournir encore davantage des informations sur le phénomène de l'assimilation vocalique régressive.

À côté des adjectifs, des postpositions, des adverbes, du morphème du pluriel et du morphème distributif, nous examinerons aussi le comportement d'autres conjonctions et particules. On abordera aussi d'autres cas particuliers d'assimilation vocalique régressive qui n'avaient pas été pris en compte dans l'article précédent.

Pour les commentaires et les remarques sur certaines catégories de mots, on se référera aux différents chapitres les concernant.

La description concernera toujours seulement les cas où les deux mots consécutifs présentent des voyelles différentes.

La langue qui fait l'objet de cette contribution s'appelle bámánánkán. Les locuteurs se nomment bámánán. Elle est plus connue sous le nom bambara. Le bamanankan est la

${ }^{1}$ Cet article revise et complète celui qui a été déjà publié dans Annali 64 sous le titre «L'assimilation vocalique régressive en bamanankan: d'autres cas». 
langue la plus importante parmi les 13 langues reconnues officiellement comme langues nationales de la République du Mali. Le bamanankan appartient au Manding, un groupe des langues Mande, une sous-famille du Niger-Congo.

\section{ADJECTIFS À TON BAS ET À TON HAUT EN $1^{\text {ÈRE }}$ POSITION}

Les adjectifs dont il sera question ici sont ceux-là qui apparaissent exclusivement après les morphèmes ká et mán et qui sont appelés par certains linguistes comme CREISSELS (1985) «verbes statifs». Notre but n'est pas de reprendre ici cette discussion, mais plutôt de décrire leur comportement quant à la problématique de l'assimilation, puisqu' ils forment à coup sûr une catégorie à part quelle que soit l'étiquette qu'on leur colle. Dans le parler décrit ici, ils sont à notre connaissance au nombre de 41 (compte tenu de la variante d'un des adjectifs) dont il ne sera tenu compte dans cet exposé que de 40. L'adjectif kùnbà 'gros, énorme' avait été exclu à cause de son statut de composé. Il faut signaler que, malgré ce statut, il ne se prête pas du tout à l'assimilation.

\subsection{ADJECTIFS À TON BAS EN 1 ÈRE POSITION:}

\subsubsection{Adjectifs à ton bas en 1ère position - pronoms en 2ème position:}

Quand les adjectifs à ton bas sont en première position et les pronoms en deuxième position, on constate que l'assimilation a lieu partout quel que soit le pronom en deuxième position, mais dans le cas de la voyelle finale $u$, elle sera accompagnée, à l'exception de l'adjectif nùkù, de la présence de l'approximant $w$, si le pronom est $a, e$ ou $i$ :

\footnotetext{
à ká wùsà í mà > [à ká w’sì: mâ:] ${ }^{2}$ c'est mieux pour toi

à ká kìsè à yé > [à ká k’ sá:!jé] $]^{3}$ il est plus courageux que lui

à ká nùkù à mà $>$ [à ká nùká: mà] c'est lisse pour lui

à ká kèkù à yé > [à ká kèkwá:!jé] il est plus malin que lui
}

\subsubsection{Adjectifs à ton bas en $1^{\text {ère }}$ position - noms propres en $2^{\text {ème }}$ position:}

Quand les adjectifs à ton bas sont en première position et les noms propres en deuxième position, on constate que:

- Lorsque la voyelle initiale du nom propre est $a$, l'assimilation a lieu partout quelle que soit la voyelle finale de l'adjectif. Mais si cette dernière est $u$, l'assimilation ne sera pas accompagnée de la présence de l'approximant $w$ seulement chez nùkù:

à ká nì àlî yé > [à ká na:lî: jé] ${ }^{4}$ il est plus gentil qu’Ali

${ }^{2}$ Dans la réalisation phonétique les voyelles peuvent tomber, mais les tons restent flottants. La suppression pure et simple de ces tons flottants entraînera une fausse réalisation phonétique.

${ }^{3}$ Dans la réalisation phonétique le '!' signifie qu'un ton haut est rabaissé de son niveau normal par un ton bas flottant, pendant que ce ton bas flottant ne se manifeste pas du tout quand il s'agit d'un ton bas qui suit.

${ }_{4}$ Toute voyelle longue sans ton dans la réalisation phonétique a la structure tonale bas-haut-bas et ne sera pas par conséquent marquée. Nous avions voulu marquer le ton à la main, mais cela surcharge inutilement l'écriture et il y'a aussi le risque d'en oublier. Nous pouvions marquer à la machine la structure tonale bas-haut, mais le deuxième bas sera flottant, ce qui ne serait pas normal car il appartient à la voyelle longue. C'est pourquoi, nous avons décidé de la laisser ici et ailleurs comme telle. 
à ká nùkù àlî mà > [à ká nùkâ:lî: mâ:] c'est lisse pour Ali

à ká kùmù àlî mà > [à ká k’mwâ:lî: mâ:] c'est amer pour Ali

- Lorsque la voyelle initiale est $i$, l'assimilation a lieu en général:

à ká wùsà ísá mà > [à ká w`sì:sá mâ:] [à ká w’sàisá mâ:] c'est mieux pour Issa

à ká kòrò ísá yé > [à ká kòrì:sá jé] il est plus âgé qu'Issa

à ká kèkù ísá yé > [à ká kèkwì:sá jé] il est plus malin qu'Issa

- Lorsque la voyelle initiale est $u$, il n'y a pas d'assimilation possible:

à ká kòrò úmù yé > [à ká kòròúm jé] il est plus âgé qu'Oumou

à ká wùsà úmù mà > [à ká w’sàúm mâ:] c'est mieux pour Oumou

\subsubsection{Adjectifs à ton bas en $1^{\text {ère }}$ position - mots étrangers en $2^{\text {ème }}$ position:}

Quand les adjectifs à ton bas sont en première position et les mots étrangers en deuxième position, on constate que l'assimilation n'a lieu que lorsque la voyelle initiale du mot étranger est $a$. Et dans cette condition, les adjectifs ont le même comportement que lorsqu'ils sont en présence de la voyelle initiale $a$ d'un nom propre (cf. 1.1.2 ci-dessus):

à ká nùkù àràjòdílálâ mà > [à ká nùkâ:ràłòd'lálá: mà] c'est lisse pour le réparateur de radio à ká kèkù àràjòdílálâ yé > [à ká kèkwâ:ràjòd'lálá:!jé] il est plus malin que le réparateur de radio à ká nì ègìlìî yé > [à kán ‘èg 'lìzí:!jé] ç’est plus joli que l’église

\subsection{ADJECTIFS À TON HAUT EN 1 ĖRE POSITION:}

\subsubsection{Adjectifs à ton haut, en $1^{\text {ère }}$ position - pronoms en $2^{\text {ème }}$ position:}

Quand les adjectifs à ton haut sont en première position et les pronoms en deuxième position, on fait le constat suivant:

- Lorsque la voyelle finale de l'adjectif est $a$, l'assimilation a lieu chez cá quel que soit le pronom en deuxième position. Quand l'adjectif est kúná, l'assimilation n'a pas lieu si le pronom est $u$; si le pronom est $i$, l'assimilation peut ne pas avoir lieu. Quant à súmá, le dernier de ce groupe, l'assimilation a lieu en général seulement si le pronom est $e$ ou $o$; dans le cas de $i$ l'assimilation reste possible:

à ká cá ù mà > [à ká cû: mâ:] c'est trop pour eux

à ká kúná í mà > [à ká kúní: mâ:] [ [à ká kúnáí mâ:] c'est amer pour toi

à ká súmá í mà > [à ká s'máí mâ:] [à ká s'mí: mâ:] c'est frais pour toi

à ká súmá ò mà > [à ká s'mô mâ:] [à ká s'máò mâ:] c'est frais pour celui-là

- Lorsque la voyelle finale est $\varepsilon$, l'assimilation a lieu généralement partout quel que soit le pronom en jeu, mais avec la remarque que l'assimilation n'a en général pas lieu chez jé si le pronom est $o$ ou $u$ :

à ká kéné ù yé > [à ká kénû: jé] il est plus sain qu'eux

à ká jé ò yé > [à ká féò jé] [à ká fô:!!jé] il est plus clair que celui-là

- Lorsque la voyelle finale est $i$, l'assimilation est observée partout:

à ká télí à yé > [à ká télá:!jé] il est plus rapide que lui

à ká télí ù yé > [à ká télû: jé] il est plus rapide qu'eux 
- Lorsque la voyelle finale est $o$, on a affaire au seul adjectif $k o ́$ chez lequel l'assimilation a lieu quel que soit le pronom en jeu. Mais l'approximant $w$ sera présent partout sauf si le pronom est $u$ :

à ká kó à yé > [à ká kwá:!jé] ça ne lui plait pas

à ká kó ù yé > [à ká kû: jé] ça ne leur plait pas

- Lorsque la voyelle finale est $っ$, on a affaire aussi au seul adjectif dókó qui admet l'assimilation partout quel que soit le pronom en deuxième position:

à ká dókj́ à mà > [à ká dóká: mà] c'est petit pour lui

à ká dókó ù mà > [à ká dókû: mâ:] c'est petit pour eux

- Lorsque la voyelle finale de l'adjectif est $u$, il s'agira du seul adjectif júkú. Là, l'assimilation a lieu avec tous les pronoms en jeu. L'approximant $w$ peut apparaître si le pronom est $a$ :

à ká júkú à mà > [à ká fúká: mà] [à ká fúkwá: mà] il est méchant à son égard

à ká júkú ò mà > [à ká fúkô: mâ:] il est méchant à l'égard de celui-là

\subsubsection{Adjectifs à ton haut en $1^{\text {ère }}$ position - noms propres en $2^{\text {ème }}$ position:}

Quand les adjectifs à ton haut sont en première position et les noms propres en deuxième position, on peut faire l'observation suivante:

- Lorsque la voyelle initiale du nom propre est $a$, l'assimilation a lieu quelle que soit la voyelle finale de l'adjectif. Mais la présence de l'approximant $w$ sera obligatoire si la voyelle finale de l'adjectif est $o$ et facultative si elle est $u$ :

à ká kéné àlî yé > [à ká kénâ:lî: jé] il est plus sain qu’Ali

à ká kó àlî yé > [à ká kwâ:lî: jé] ça ne plait pas à Ali

à ká júkú àlî mà > [à ká júkâ:lî: mâ:] [à ká fúkwâ:lî: mâ:] il est méchant à l'égard d'Ali

- Lorsque la voyelle initiale est $i$, l'assimilation peut avoir lieu seulement chez cá si la voyelle finale de l'adjectif est $a$. Quand la voyelle finale est $\varepsilon$, l'assimilation a lieu seulement chez $k \dot{n} n \varepsilon \dot{\varepsilon}$; mais elle reste possible chez $j \varepsilon$. Quand la voyelle finale est $o$ ou $u$, l'assimilation a lieu avec l'approximant $w$. Mais dans le cas de $u$, l'assimilation peut ne pas être observée. Quand la voyelle finale de l'adjectif est o, l'assimilation a lieu en général:

\footnotetext{
à ká cá ísá mà > [à ká cáisá mâ:] [à ká cí:sá mâ:] c'est trop pour Issa

à ká kúná ísá mà > [à ká kúnáísá mâ:] c'est amer pour Issa

à ká kéné ísá yé > [à ká kéní:sá jé] il est plus sain qu'Issa

à ká kó ísá yé > [à ká kwí:sá jé] ça ne plait pas à Issa

à ká dókó ísá mà > [à ká dókí:sá mâ:] [à ká dókóísá mâ:] c’est petit pour Issa

- Il n'y a pas d'assimilation, lorsque la voyelle initiale du nom propre est $u$ :

à ká kúná úmù mà $>$ [à ká kúnáúm mâ:] c'est amer pour Oumou

à ká kó úmù yé > [à ká kóúm jé] ça ne plait pas à Oumou
}

1.2.3. Adjectifs à ton haut en $1^{\text {ère }}$ position - mots étrangers en $2^{\text {ème }}$ position:

Quand les adjectifs à ton haut sont en première position et les mots étrangers en deuxième position, on constate que l'assimilation n'a lieu que lorsque la voyelle initiale du mot 
étranger est $a$. Dans cette condition les adjectifs ont le même comportement que devant la voyelle initiale $a$ du nom propre (cf. plus haut):

à ká dóḱ àràjòdílálâ mà > [à ká dókâ:ràjòd'lálá: mà] c'est petit pour le réparateur de radio

à ká cá ìzínî báárâ mà > [à ká cáizíń!bá:rá: mà] c'est trop pour le travail à l'usine

\section{ADJECTIFS NASALISÉS À TON BAS ET À TON HAUT EN $1^{\text {ĖRE }}$ POSITION}

\subsection{ADJECTIFS NASALISÉS À TON BAS EN $1{ }^{\text {ĖRE }}$ POSITION}

\subsubsection{Adjectifs nasalisés à ton bas en $1^{\text {ère }}$ position - pronoms en $2^{\text {ème }}$ position}

Quand les adjectifs nasalisés à ton bas sont en première position et les pronoms en deuxième position, force est de constater que:

- Lorsque la voyelle finale de l'adjectif est an, l'assimilation peut être observée chez jàn et seulement si le pronom est $e, i$ ou $o$ :

à ká jàn í yé > [à ká fàì jé] [à ká jì: jé] il est plus grand que toi

à ká jàn ù yé $>$ [à ká fàù jé] il est plus grand qu'eux

- Lorsque la voyelle finale est en, on a affaire au seul adjectif bilèn qui perd sa nasalité et admet l'assimilation partout sauf si le pronom est $u$ :

à ká bìlèn à yé > [à ká b 'lá:!jé] il est plus rouge que lui

à ká bìlèn ù yé > [à ká b 'éù jé] il est plus rouge qu'eux

- Lorsque la voyelle finale de l'adjectif est $\varepsilon n$, la nasalité tombe partout et l'assimilation a lieu quel que soit le pronom en jeu:

à ká gè lèn à mà > [à ká gè lá: mà] c'est difficile pour lui

- Lorsque la voyelle finale est in, il s'agira du seul adjectif fin dont la nasalité est stable. Il participe à l'assimilation où l'approximant $j$ apparaît:

à ká fìn à yé > [à ká fjặ:!jé] il est plus noir que lui

à ká fîn ò yé > [à ká fjo: jé] 〜 [à ká fĩò jé] il est est plus noir que celui-là

- Lorsque la voyelle finale de l'adjectif est $u n$, l'assimilation a lieu partout, mais elle sera accompagnée de la présence de l'approximant $w$ chez dùn qui conserve le plus souvent sa nasalité contrairement à sùrùn où la nasalité disparaît complètement:

à ká dùn à mà > [à ká dwă̌: mà] [à ká dwă: mà] c'est profond pour lui

à ká dùn ò mà < [à ká dwo: mâ:] c'est profond pour celui-là

à ká sùrùn ò mà $>$ [à ká sùrô: mâ:] c'est court pour celui-là

- Lorsque la voyelle finale de l'adjectif est on, on aura affaire au seul adjectif bòn qui perd sa nasalité et participe en général à l'assimilation quel que soit le pronom en deuxième position. Mais l'approximant $w$ sera présent si le pronom est $a, e$ ou $i$ :

à ká bòn à mà > [à ká bwă: mà] c'est grand pour lui

à ká bòn ù mà $>$ [à ká bù: mâ:] [à ká bòù mâ:] c'est grand pour eux 


\subsubsection{Adjectifs nasalisés à ton bas en $1^{\text {ère }}$ position - noms propres en $2^{\text {ème }}$ position}

Quand les adjectifs nasalisés à ton bas sont en première position et les noms propres en deuxième position, on constate que:

- Lorsque la voyelle initiale du nom propre est $a$, l'assimilation n'a pas lieu en général quand la voyelle finale de l'adjectif est en; dans les autres cas, elle a lieu; mais elle sera accompagnée de l'apparition de l'approximant $w$ si l'adjectif est bòn et dùn, et de l'approximant $j$ si l'adjectif est fin:

\footnotetext{
à ká bìlèn àlî yé > [à ká b 'léàlî: jé] [à ká b`lâ:lî: jé] il est plus clair qu’Ali

à ká fìn àlî yé > [à ká fjạ:lî: jé] il est plus noir que lui

à ká bòn àlî mà > [à ká bwa:lî: mâ:] c'est grand pour Ali
}

- Lorsque la voyelle initiale est $i$, l'assimilation n'a pas lieu en général quand la voyelle finale de l'adjectif est an ou en. Quand la voyelle finale de l'adjectif est $\varepsilon n$, l'assimilation aura lieu seulement si l'adjectif est gèlèn. Quand elle est on, l'assimilation a lieu avec la présence de l'approximant $w$. Quand la voyelle finale de l'adjectif est un, l'assimilation a lieu, mais elle sera accompagnée de l'apparition de l'approximant $w$ si l'adjectif est dùn:

\footnotetext{
à ká jàn ísá yé > [à ká jàísá jé] [à ká jì:sá jé]il est plus grand qu’Issa

à ká gè lèn ísá mà > [à ká gè lì:sá mâ:] c'est difficile pour Issa

à ká bòn ísá mà > [à ká bwì:sá mâ:] [à ká bwòísá mâ:] c'est grand pour Issa

à ká dùn ísá mà > [à ká dwì:sá mâ:] c'est profond pour Issa

- Lorsque la voyelle initiale du nom propre est $u$, l'assimilation n'a lieu nulle part:

à ká jàn úmù yé > [à ká fàúm jé] il est plus grand qu'Oumou

à ká gè lèn úmù mà > [à ká gè lèúm mâ:] c'est difficile pour Oumou
}

\subsubsection{Adjectifs nasalisés à ton bas en $1{ }^{\text {ère }}$ position - mots étrangers en $2^{\text {ème }}$ position}

Quand les adjectifs nasalisés à ton bas sont en première position et les mots étrangers en deuxième position, on constate que l'assimilation n'a lieu que lorsque la voyelle initiale du mot étranger est $a$. Dans ce cas, le comportement des adjectifs est identique à celui qu'ils ont devant la voyelle initiale $a$ du nom propre (cf. 2.1 .2 ci-dessus):

à ká gè lèn àràjô yé > [à ká gè lâ:ràjó:!jé] c'est plus cher que la radio

à ká bòn àràjòdílálâ mà > [à ká bwa:ràjòd'lálá: mà] c'est grand pour le réparateur de radio

à ká sùrùn ìzínî lá > [à ká sùrúizíń!ná] ce n'est pas loin de l’usine

\subsection{ADJECTIFS NASALISÉS À TON HAUT EN $1^{\text {ĖRE }}$ POSITION}

\subsubsection{Adjectifs nasalisés à ton haut en $1^{\text {ère }}$ position - pronoms en $2^{\text {ème }}$ position}

Quand les adjectifs nasalisés à ton haut sont en première position et les pronoms en deuxième position, on constate la chose suivante:

- Lorsque la voyelle finale de l'adjectif est an, l'assimilation peut avoir lieu si le pronom est $e$ :

à ká kán ê yé > [à ká kạ́é:!jé] [à ká kế:!jé] ça t’est égal

à ká kán ù yé > [à ká kạuu jé] ça leur est égal 
- Lorsque la voyelle finale est in et on, l'assimilation a lieu avec tous les pronoms en deuxième position:

à ká gírín à mà > [à ká gírá: mà] c’est lourd pour lui

à ká kólón ù yé > [à ká kólû: jé] il est plus paresseux qu'eux

\subsubsection{Adjectifs nasalisés à ton haut en $1^{\text {ère }}$ position - noms propres en $2^{\text {ème }}$ position}

Quand les adjectifs nasalisés à ton haut sont en première position et les noms propres en deuxième position, on peut faire l'observation suivante:

- Lorsque la voyelle initiale du nom propre est $a$, l'assimilation a lieu quelle que soit la voyelle finale de l'adjectif en première position:

à ká gírín àlî mà > [à ká gírâ:lî: mâ:] c'est lourd pour Ali

- Lorsque la voyelle initiale du nom propre est $i$, l'assimilation a lieu en général seulement si l'adjectif est kólón:

à ká kólón ísá yé > [à ká kólí:sá jé] [à ká kólóísá jé] il est plus paresseux qu'Issa

- Lorsque la voyelle initiale du nom propre est $u$, aucune assimilation n'a lieu:

à ká kán úmù yé > [à ká kấúm jé] c'est égal pour Oumou

2.2.3. Adjectifs nasalisés à ton haut en $1^{\text {ère }}$ position - mots étrangers en $2^{\text {ème }}$ position

Quand les adjectifs nasalisés à ton haut sont en première position et les mots étrangers en deuxième position, on constate que l'assimilation aura lieu seulement si la voyelle initiale du mot étranger est $a$. Dans ce cas, les adjectifs ont le même comportement que lorsqu'ils sont en présence de la voyelle initiale $a$ du nom propre:

à ká gírín àràjô yé > [à ká gírâ:ràjó:!jé] c'est plus lourd que la radio

à ká kán ègìlizî báárákélâ yé > [à ká kạàg 'lizí:!bá:rákélá:!jé] c'est égal pour l’ouvrier de l'église

\section{LES POSTPOSITIONS ET LES NOMINAUX FAISANT FONCTION DE POSTPOSITION EN 1 ĖRE POSITION}

\subsection{LA POSTPOSITION kàn EN 1 ĖRE POSITION}

\subsubsection{La postposition kàn en $1^{\text {ère }}$ position - pronoms en $2^{\text {ème }}$ position}

Quand la postposition kàn est en première position et les pronoms en deuxième position, on constate que l'assimilation n'est pas observée. Il faut signaler en passant que la postposition kàn perd sa nasalité ici et tend en général vers une dénasalisation dans tout son emploi syntaxique:

à bí à kàn í kóró yèn > [à bá: kàikóró jè] il est là-dessus là-bas à côté de toi

à bí à kàn ò kóró yèn > [à bá: kàò kóró jè] il est là-dessus là-bas à côté de celui-là 
3.1.2. La postposition kàn en $1^{\text {ère }}$ position - noms propres en $2^{\text {ème }}$ position

Quand la postposition kàn est en première position et les noms propres en deuxième position, on fait le même constat que sous 3.1.1 ci-dessus:

à bí à kàn ísá kóró yèn > [à bá: kàisá kóró jè] il est là-dessus là-bas à côté d'Issa

à bí à kàn úmù kóró yèn > [à bá: kàúm kóró jè] il est là-dessus là-bas à côté d'Oumou

3.1.3. La postposition kàn en $1^{\text {ère }}$ position - mots étrangers en $2^{\text {ème }}$ position

Quand la postposition kàn est en première position et les mots étrangers en deuxième position, on fait le même constat que sous 3.1.1 et 3.1.2 ci-dessus:

à bí à kàn è rè gílî kóró yèn > [à bá: kàè rè g'lí:!kóró jè] c'est là-dessus là-bas à côté de la règle

à bí à kàn úrúsî kóró yèn > [à bá: kàúr' sí:!kóró jè] il est là-dessus là-bas à côté de l'ours

\subsection{LA POSTPOSITION yé EN $1^{\text {ÈRE }}$ POSITION}

3.2.1. La postposition yé en $1^{\text {ère }}$ position - pronoms en $2^{\text {ème }}$ position

Quand la postposition yé est en première position et les pronoms en deuxième position, on constatera que l'assimilation a lieu partout sans exception:

à má fó à yé à nê lá > [à má fwá:!já:!nć:!ná] on ne lui a pas dit en sa présence

à má fó à yé ò nê lá > [à má fwá:!jô: né:!ná] on ne lui a pas dit en présence de celui-là

\subsubsection{La postposition yé en $1^{\text {èr }}$ position - noms propres en $2^{\text {ème }}$ position}

Quand la postposition yé est en première position et les noms propres en deuxième position, on peut faire l'observation suivante:

- Lorsque la voyelle initiale du nom propre est $a$ ou $i$, l'assimilation a lieu:

à má fỏ à yé àlî nê lá > [à má fwá:!jâ:lî: né:!ná] on ne lui a pas dit en présence d'Ali

- Par contre, lorsque la voyelle initiale du nom propre est $u$, toute possibilité d'assimilation est exclue:

à má fó à yé úmù jê lá > [à má fwá:!jéúm̀ né:!ná] on ne lui a pas dit en présence d'Oumou

\subsubsection{La postposition yé en $1^{\text {ère }}$ position - mots étrangers en $2^{\text {ème }}$ position}

Quand la postposition yé est en première position et les mots étrangers en deuxième position, force est de constater que, lorsque la voyelle initiale du mot étranger est seulement $a, \varepsilon$ ou $ว$, l'assimilation est observée:

à má f’̉ à yé èrè gílî káríbáâ nê lá > [à má fwá:!jê:rè g'lí:!kár'bá:!né:!ná] on ne lui a pas dit en présence de celui qui a cassé la règle

à má f’’ à yé ìzínî báárákélâ nê lá > [à má fwá:!jéizińn!bá:rákélá:!né:!ná] on ne lui a pas dit en présence de l'ouvrier de l'usine

\subsection{A POSTPOSITION $f \dot{\varepsilon}$ EN $1{ }^{\text {ĖRE }}$ POSITION}

3.3.1. La postposition $f \dot{\varepsilon}$ en $1^{\text {ère }}$ position - pronoms en $2^{\text {ème }}$ position

Quand la postposition $f \dot{\varepsilon}$ est en première position et les pronoms en deuxième position, on fait le constat suivant: 
- Lorsque le pronom est $a, o$ ou $u$, toute possibilité d'assimilation est exclue:

à bí à fè à ká táá > [à bá: fè à ká tá:] il veut qu'il parte

à bí à fè ù ká táá > [à bá: fèù ká tá:] il veut qu'ils partent

- Lorsque le pronom est $e$ ou $i$ l'assimilation a en général lieu:

à bí à fề í ká táá > [à bá: fì: ká tá:] [à bá: fè̀ ká tá:] il veut que tu partes

\subsubsection{La postposition $f \dot{\varepsilon}$ en $1^{\text {ère }}$ position - noms propres en $2^{\text {ème }}$ position}

Quand la postposition $f \hat{\varepsilon}$ est en première position et les noms propres en deuxième position, force est de constater que sa voyelle finale ne s'assimile à aucune voyelle initiale du nom propre sauf si elle est $i$, où l'assimilation est possible:

à bí à fè àlî ká táá > [à bá: fèàlî: ká tá:] il veut qu'Ali parte

à bí à fè ísá ká táá > [à bá: fêísá ká tá:] [à bá: fî:sá ká tá:] il veut qu'Issa parte

\subsubsection{La postposition $f \dot{\varepsilon}$ en $1^{\text {ère }}$ position - mots étrangers en $2^{\text {ème }}$ position}

Quand la postposition $f \dot{\varepsilon}$ est en première position et les mots étrangers en deuxième position, on constate que sa voyelle finale ne s'assimile à aucune voyelle initiale du mot étranger:

à bí à fè àràjòdílálâ ká táá > [à bá: fè̀àà̀òd'lálá:!ká tá:] il veut que le réparateur de radioparte

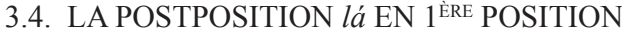

\subsubsection{La postposition lá en $1^{\text {ère }}$ position - pronoms en $2^{\text {ème }}$ position}

Quand la postposition lá est en première position et les pronoms en deuxième position, l'assimilation a lieu:

à má màkà à lá ê jê lá > [à má màká:!lé:!nć:!ná] il ne l'a pas touché devant toi

à má màkà à lá ò nê lá > [à má màká:!lô: né:!ná] il ne l'a pas touché devant celui-là

\subsubsection{La postposition lá en $1^{\text {ère }}$ position - noms propres en $2^{\text {ème }}$ position}

Quand la postposition lá est en première position et les noms propres en deuxième position, on constate que l'assimilation a lieu en général seulement, si la voyelle initiale du nom propre est $i$ :

à má màkà à lá ísá nê lá > [à má màká:!lí:sá né:!ná] [à má màká:!láísá né:!ná] il ne l'a pas touché devant Issa

à má màkà à lá úmù nê lá > [à má màká:!láúm né:!ná] il ne l’a pas touché devant eux

\subsubsection{La postposition lá en $1^{\text {ère }}$ position - mots étrangers en $2^{\text {ème }}$ position}

Quand la postposition lá est en première position et les mots étrangers en deuxième position, on constate que l'assimilation n'a pas lieu:

à má màkà à lá ègìlìî kónó > [à má màká:!láèg 'lìzí:!kónó] il ne l'a pas touché dans l'église 


\subsection{LA POSTPOSITION kámà EN 1 ĖRE POSITION}

3.5.1. La postposition kámà en $1^{\text {ère }}$ position - pronoms en $2^{\text {ème }}$ position

Quand la postposition kámà est en première position et les pronoms en deuxième position, force est de constater que l'assimilation a lieu partout sans exception:

à tááá à kámà ù ká só > [à tá:rá:!kámù: ká só] il est allé chez eux pour l'attaquer

\subsubsection{La postposition kámà en $1^{\text {ère }}$ position - noms propres en $2^{\text {ème }}$ position}

Quand la postposition kámà est en première position et les noms propres en deuxième position, on constate que l'assimilation n'a lieu nulle part:

à tááá à kámà úmù ká só > [à tá:rá:!kámàúm ká só] il est allé chez Oumou pour l’attaquer

\subsubsection{La postposition kámà en $1^{\text {ère }}$ position - mots étrangers en $2^{\text {ème }}$ position}

Quand la postposition kámà est en première position et les mots étrangers en deuxième position, on constate que l'assimilation n'est observée nulle part:

à táárá à kámà érésítórân kónó > [à tá:rá:!kámàćrés' tóráá:!kónó] il est allé au restaurant pour l'attaquer

\subsection{LE NOMINAL kónó FAISANT FONCTION DE POSTPOSITION EN 1 ĖRE POSITION}

3.6.1. Le nominal kónó faisant fonction de postposition en $1^{\text {ère }}$ position - pronoms en $2^{\text {ème }}$ position

Quand le nominal kónó faisant fonction de postposition est en première position et les pronoms en deuxième position, force est de constater que l'assimilation a lieu avec tous les pronoms sans exception:

à bí à kónó à kóró yèn > [à bá:!kóná:!kóró jè] c'est là-dedans là-bas à côté de lui

à yé à bìlà à kónó ù kóró yèn > [à já: b` lá:!kónû: kóró jè] il l'a mis là-dedans là-bas à côté d'eux

3.6.2. Le nominal kónó faisant fonction de postposition en $1^{\text {ère }}$ position - noms propres en $2^{\text {ème }}$ position

Quand le nominal kónó faisant fonction de postposition est en première position et les noms propres en deuxième position, on peut faire l'observation suivante:

- Lorsque la voyelle initiale du nom propre est $a$ ou $i$, l'assimilation est observée:

à bí à kónó àlî kóró yèn > [à bá:!kónâ:lî: kóró jè] c'est là-dedans là-bas à côté d'Ali

- Lorsque la voyelle initiale du nom propre est $u$, toute possibilité d'assimilation est exclue:

à yé à bìlà à kónó úmù kóró yèn > [à já: b lá:!kónóúm kóró jè] il l'a mis là-dedans là-bas à côté d'Oumou 
3.6.3. Le nominal kónó faisant fonction de postposition en $1^{\text {ère }}$ position - mots étrangers en $2^{\text {ème }}$ position

Quand le nominal kónó faisant fonction de postposition est en première position et les mots étrangers en deuxième position, on constate que l'assimilation a lieu seulement si la voyelle initiale du mot étranger est $a$ :

à bí à kónó àràjô kóró yèn > [à bá:!kónâ:ràfó:!kóró jè] c'est là-dedans là-bas à côté de la radio à yé à bilà à kónó ègìlizî dâ lá > [à já: b lá:!kónóèg lìzí:!dá:!lá] il l’a mis là-dedans devant l’église

\subsection{LE NOMINAL $m a ̀$ FAISANT FONCTION DE POSTPOSITION EN $1^{\text {ĖRE }}$ POSITION}

3.7.1. Le nominal mà faisant fonction de postposition en $1^{\text {ère }}$ position - pronoms en $2^{\text {ème }}$ position

Quand le nominal mà faisant fonction de postposition est en première position et les pronoms en deuxième position, on constate que l'assimilation n'est nulle part observée.

à bí dùkû mà í kóró yèn > [àb' dùkú: mài kóró jè] c'est là-bas par terre à côté de toi

à bí dùkû mà ù kóró yèn > [àb'dùkú: màù kóró jè] c'est là-bas par terre à côté d'eux

3.7.2. Le nominal mà faisant fonction de postposition en $1^{\text {ère }}$ position - noms propres en $2^{\text {ème }}$ position

Quand le nominal mà faisant fonction de postposition est en première position et les noms propres en deuxième position, le constat est le même que lorsque les pronoms sont en deuxième position (cf. 3.7.1 ci-dessus):

à bí dùkû mà ísá kóró yèn > [àb'dùkú: màísá kóró jè] c'est là-bas par terre à côté d'Issa

à bí dùkû mà úmù kóró yèn > [àb' dùkú: màúm kóró jè] c'est là-bas par terre à côté d'Oumou

3.7.3. Le nominal mà faisant fonction de postposition en $1^{\text {ère }}$ position - mots étrangers en $2^{\text {ème }}$ position

Quand le nominal mà faisant fonction de postposition est en première position et les mots étrangers en deuxième position, on fait le même constat que sous 3.7 .1 et 3.7.2 cidessus:

à bí dùkû mà ègìlìî kónó yèn > [àb' dùkú: màèg 'lìzí:!kónó jè] c'est là-bas par terre dans l'église à bí dùkû mà ìzínî kónó yèn > [àb'dùkú: màizíńkónó jè] c'est là-bas par terre dans l'usine

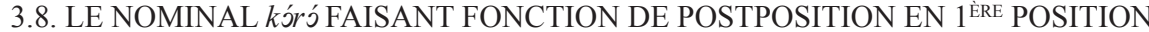

3.8.1. Le nominal kóró faisant fonction de postposition en $1^{\text {ère }}$ position - pronoms en $2^{\text {ème }}$ position

Quand le nominal kóró faisant fonction de postposition est en première position et les pronoms en deuxième position, on constatera que l'assimilation a lieu partout:

à bí jírî kóró à né yèn > [àb' jírí:!kórá:!né jè] il est sous l'arbre à l'attendre là-bas

à bí jírî kóró ò né yèn > àb' jírí:!kórô: né jè] il est sous l'arbre à attendre celui-là là-bas 
3.8.2. Le nominal kóró faisant fonction de postposition en $1^{\text {ère }}$ position - noms propres en $2^{\text {ème }}$ position

Quand le nominal kóró faisant fonction de postposition est en première position et les noms propres en deuxième position, on constate la chose suivante:

- Lorsque la voyelle initiale du nom propre est a ou $i$, l'assimilation a lieu en général:

à bí jírî kóró àlî né yèn > [àb'jíríí:!kórâ:lî: né jè] il est sous l'arbre à attendre Ali là-bas

à bí jírî kóró ísá né yèn > [àb 'jírí:!kórí:sá né jè] _[àb' jírí:!kóróísá né jè] il est sous l'arbre à attendre Issa là-bas

- Toute possibilité d'assimilation reste exclue, lorsque la voyelle initiale du nom propre est $u$ :

à bí jírî kóró úmù né yèn > [àb'fíríi:!kóróúm né jè] il est sous l'arbre à attendre Oumou là-bas

3.8.3. Le nominal kóró faisant fonction de postposition en $1^{\text {ère }}$ position - mots étrangers en $2^{\text {ème }}$ position

Quand le nominal kóró faisant fonction de postposition est en première position et les mots étrangers en deuxième position, l'assimilation aura lieu seulement si la voyelle initiale du mot étranger est $a$ :

à bí jírî kóró àràjòdílálâ né yèn > [àb'jírí:!kórâ:ràjòd'lálá:!né jè] il est sous l'arbre à attendre le réparateur de radio là-bas

à bí jírî kóró ègilìzî dâ lá yèn > [àb'jírí:!kóróèg 'lìzí:!dá:!lá jè] il est sous l'arbre devant l’église là-bas

\subsection{LE NOMINAL $n \varepsilon ́$ FAISANT FONCTION DE POSTPOSITION EN $1{ }^{\text {ĖRE }}$ POSITION}

3.9.1. Le nominal $n \varepsilon ́$ faisant fonction de postposition en $1^{\text {ère }}$ position - pronoms en $2^{\text {ème }}$ position

Quand le nominal né faisant fonction de postposition est en première position et les pronoms en deuxième position, on peut observer que l'assimilation a lieu partout:

à bí í né à ká só yèn > [à bí: ná:!ká só jè] il t'attend chez lui là-bas

à bí í né ò ká só yèn > [à bí: nô: ká só jè] il t'attend chez celui-là là-bas

3.9.2. Le nominal $n \varepsilon ́$ faisant fonction de postposition en $1^{\text {ère }}$ position - noms propres en $2^{\text {ème }}$ position

Quand le nominal $n \varepsilon ́$ faisant fonction de postposition est en première position et les noms propres en deuxième position, force est de constater que l'assimilation n'a lieu que si la voyelle initiale du nom propre est $a$ :

à bí í né àlî ká só yèn > [à bí: nâ:lî: ká só jè] il t’attend chez Ali là-bas

à bí í né úmù ká só yèn > [à bí: néúm ká só jè] il t’attend chez Oumou là-bas

3.9.3. Le nominal $n \varepsilon ́$ faisant fonction de postposition en $1^{\text {ère }}$ position - mots étrangers en $2^{\text {ème }}$ position

Quand le nominal $n \varepsilon$ faisant fonction de postposition est en première position et les 
mots étrangers en deuxième position, on constate que l'assimilation n'a lieu ici aussi que lorsque la voyelle initiale du mot étranger est $a$ :

à bí í né àràjòdílálâ ká só yèn > [à bí: nâ:ràjòd'lálá:!ká só jè] il t’attend chez le réparateur de radio là-bas à bí à né ègìlìîi dâ lá yèn > [à bá:!nćèg lìzí:!dá:!lá jè] il l’attend devant l'église là-bas

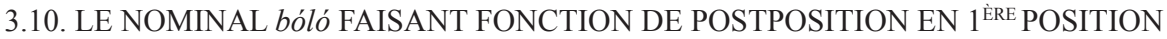

3.10.1. Le nominal bóló faisant fonction de postposition en $1^{\text {ère }}$ position - pronoms en $2^{\text {ème }}$ position

Quand le nominal bóló faisant fonction de postposition est en première position et les pronoms en deuxième position, on constate que l'assimilation a lieu partout sans exception:

à bí à bóló à ká só > [à bá:!bólá:!ká só] il l'a chez lui

à bí à bóló ù ká só > [à bá:!bólû: ká só] il l'a chez eux

3.10.2. Le nominal bóló faisant fonction de postposition en $1^{\text {ere }}$ position - noms propres en $2^{\text {ème }}$ position

Quand le nominal bóló faisant fonction de postposition est en première position et les noms propres en deuxième position, on peut constater la chose suivante:

- Lorsque la voyelle initiale du nom propre est $a$, l'assimilation est observée, et elle a lieu en général, lorsque la voyelle initiale est $i$ :

à bí à bóló àlî ká só > [à bá:!bólâ:lî: ká só] il l'a chez Ali

à bí à bóló ísá ká só > [à bá:!bólí:sá ká só] [à bá:!bólóísá ká só] il l'a chez Issa

- Par contre il n'y a pas d'assimilation possible, lorsque la voyelle initiale du nom propre est $u$ :

à bí à bóló úmù ká só > [à bá:!bóloúum ká só] il l'a chez Oumou

3.10.3. Le nominal bóló faisant fonction de postposition en $1^{\text {ère }}$ position - mots étrangers en $2^{\text {ème }}$ position

Quand le nominal bóló faisant fonction de postposition est en première position et les mots étrangers en deuxième position, on constatera que l'assimilation a lieu seulement si la voyelle initiale du mot étranger est $a$ :

à bí à bóló àràjô kóró > [à bá:!bólâ:ràjó:!kóró] il l'a à côté de la radio

à bí à bóló ègìlizî kónó > [à bá:!bólóèg lìzí:!kónó] il l'a dans l'église

\subsection{LE NOMINAL kùn FAISANT FONCTION DE POSTPOSITION EN $1^{\text {ĖRE }}$ POSITION}

3.11.1. Le nominal kùn faisant fonction de postposition en $1^{\text {ère }}$ position - pronoms en $2^{\text {ème }}$ position

Quand le nominal kùn faisant fonction de postposition est en première position et les pronoms en deuxième position, force est de constater que l'assimilation a lieu partout: 
jòlì bí ê kùn à nê lá > [jòl 'bé: kwạ̀: né:!ná] il croit que tu as combien sur toi?

jòlì bí à kùn ò nê lá > [jòl'bá: kwò̀: né:!ná] celui-là croit qu'il a combien sur lui?

3.11.2. Le nominal kùn faisant fonction de postposition en $1^{\text {ère }}$ position - noms propres en $2^{\text {ème }}$ position

Quand le nominal kùn faisant fonction de postposition est en première position et les noms propres en deuxième position, le constat qu'on peut faire est que l'assimilation a lieu seulement si la voyelle initiale du nom propre est $a$ :

jòlì bí ê kùn àlî nê lá > [jòl bé: kwậ:lî: né:!ná] Ali croit que tu as combien sur toi?

jòlì bí à kùn ísá nê lá > [jòl’bá: kù̃ísá né:!ná] Issa croit qu’il a combien sur lui?

3.11.3. Le nominal kùn faisant fonction de postposition en $1^{\text {ìre }}$ position - mots étrangers en $2^{\text {ème }}$ position

Quand le nominal kùn faisant fonction de postposition est en première position et les mots étrangers en deuxième position, on constatera que l'assimilation n' a lieu que lorsque la voyelle initiale du mot étranger est $a$ :

jòlì bí ê kùn àràjòdílálâ jê lá > [jòl 'bé: kwạ:ràjòd'lálá:!né:!ná] le réparateur de radio croit que tu as combien sur toi?

jòlì bí à kùn ègìlìzî bááákélâ nê lá > [jòl bá: kù̀èg lìzí:!bá:rákéláa! né:!ná] l’ouvrier de l'église croit qu'il a combien sur lui?

\subsection{LE NOMINAL dá FAISANT FONCTION DE POSTPOSITION EN 1 ĖRE POSITION}

3.12.1. Le nominal $d a ́$ faisant fonction de postposition en $1^{\text {ire }}$ position - pronoms en $2^{\text {ème }}$ position

Quand le nominal dá faisant fonction de postposition est en première position et les pronoms en deuxième position, on constate que l'assimilation n'est pas du tout observée:

ní à bórá à dá í jê lá > [ná:!bórá:!dáí né:!ná] si ça sort de sa bouche devant toi ... ní à bórá à dá ù nê lá > [ná:!bórá:!dáù né:!ná] si ça sort de sa bouche devant eux ...

3.12.2. Le nominal dá faisant fonction de postposition en $1^{\text {ère }}$ position - noms propres en $2^{\text {ème }}$ position

Quand le nominal dá faisant fonction de postposition est en première position et les noms propres en deuxième position, force est de constater que toute possibilité d'assimilation est exclue quelle que soit la voyelle initiale du nom propre:

ní à bórá à dá ísá nê lá > [ná:!bórá:!dáísá né:!ná] si ça sort de sa bouche devant Issa ... ní à bórá à dá úmù nê lá > [ná:!bórá:!dáúm né:!ná] si ça sort de sa bouche devant Oumou ...

3.12.3. Le nominal $d a ́$ faisant fonction de postposition en $1^{\text {ère }}$ position - mots étrangers en $2^{\text {ème }}$ position

Quand le nominal dá faisant fonction de postposition est en première position et les 
mots étrangers en deuxième position, force est de constater que toute possibilité d'assimilation est exclue aussi quelle que soit la voyelle initiale du mot étranger:

ní à bórá à dá ègilìizi báárákélâ nê lá > [ná:!bórá:!dáèg lìzí:!bá:rákélá:!né:!ná] si ça sort de sa bouche devant l'ouvrier de l'église ...

ní à bórá à dá ìzínî báárákélâ nê lá > [ná:!bórá:!dáizíń!bá:rákélá:!né:!ná] si ça sort de sa bouche devant l'ouvrier de l'usine ...

\subsection{LE NOMINAL $c \varepsilon ́$ FAISANT FONCTION DE POSTPOSITION EN 1 ĖRE POSITION}

3.13.1. Le nominal $c \varepsilon$ faisant fonction de postposition en $1^{\text {ère }}$ position - pronoms en $2^{\text {ème }}$ position

Quand le nominal cé faisant fonction de postposition est en première position et les pronoms en deuxième position, on peut constater que l'assimilation a lieu en général:

à má tílá á cé à nê lá > [à má t'lá: cá:!né:!ná] ça n’a pas été partagé entre vous devant lui

à má tílá á cé ò nê lá > [à má t'lá: cô: né:!ná] [à má t'lá: céò né:!ná] ça n’a pas été partagé entre vous devant celui-là

\subsubsection{Le nominal $c \varepsilon ́$ faisant fonction de postposition en $1^{\text {ère }}$ position - noms propres} en $2^{\text {ème }}$ position

Quand le nominal $c \varepsilon$ faisant fonction de postposition est en première position et les noms propres en deuxième position, on constatera que l'assimilation n'a lieu que lorsque la voyelle initiale du nom propre est $a$ :

à má tílá án cé àlî nê lá > [à má t'lá:njâ:lî: né:!ná] ça n'a pas été partagé entre nous devant Ali

à má tílá án cé ísá jê lá > [à má t'lá:njećísá jé:!ná] ça n'a pas été partagé entre nous devant Issa

3.13.3. Le nominal $c \varepsilon$ faisant fonction de postposition en $1^{\text {ère }}$ position - mots étrangers en $2^{\text {ème }}$ position

Quand le nominal $c \varepsilon$ faisant fonction de postposition est en première position et les mots étrangers en deuxième position, on constatera que l'assimilation n'a lieu ici aussi que lorsque la voyelle initiale du mot étranger est $a$ :

à má tílá án cé àràjòdílálâ nê lá > [à má t'lá:njâ:ràjòd'lálá:!né:!ná] ça n’a pas été partagé entre nous devant le réparateur de radio

à má tílá án cé ègilìzî báárákélâ nê lá > [à má t'lá:njéèèg lìzí:!bá:rákéláa!!nć:!ná] ça n’a pas été partagé entre nous devant l'ouvrier de l'église

\subsection{LE NOMINAL kó FAISANT FONCTION DE POSTPOSITION EN 1 ĖRE POSITION}

3.14.1. Le nominal $k$ ś faisant fonction de postposition en $1^{\text {ère }}$ position - pronoms en $2^{\text {ème }}$ position

Quand le nominal $k j$ faisant fonction de postposition est en première position et les pronoms en deuxième position, on peut observer que l'assimilation a lieu partout sans ex- 
ception. Mais elle sera accompagnée de la présence obligatoire de l'approximant w, si le pronom est $a, e$ ou $i$.

à fórá à kó í fè yèn > [à fórá:!kwí: fẽ:j'] il ne l'a pas trouvé chez toi

à fórá à kó ù fè yèn > [à fórá:!kû: fẽ:j'] il ne l'a pas trouvé chez eux

3.14.2. Le nominal $k$ j́ faisant fonction de postposition en $1^{\text {ère }}$ position - noms propres en $2^{\text {ème }}$ position

Quand le nominal kj faisant fonction de postposition est en première position et les noms propres en deuxième position, on constate que:

- Lorsque la voyelle initiale du nom propre est $a$, l'assimilation a lieu, mais avec la présence obligatoire de l'approximant $w$ :

à fórá à kó àlî fè yèn > [à fórá:!kwâ:lî: fẽ:j'] il ne l'a pas trouvé chez Ali

- Lorsque la voyelle initiale est $i$, l'assimilation n'a pas lieu en général, mais elle est possible avec l'apparition de l'approximant $w$ :

à fớrá à kó ísá fè yèn > [à fơrá:!kóísá fẽ:j'] [à fórá:!kwí:sá fẽ:j]'] il ne l’a pas trouvé chez Issa

- L'assimilation n'est pas possible, si la voyelle initiale du nom propre est $u$ :

à fórá à kó úmù fè yèn > [à fórá:!kóúm fê:j'] il ne l'a pas trouvé chez Oumou

3.14.3. Le nominal $k$ ó faisant fonction de postposition en $1^{\text {ère }}$ position - mots étrangers en $2^{\text {ème }}$ position

Quand le nominal $k$ ś faisant fonction de postposition est en première position et les mots étrangers en deuxième position, force est de constater que l'assimilation a lieu seulement si la voyelle initiale du mot étranger est $a$. Dans ce cas, l'approximant $w$ apparaît nécessairement:

à fórá à kó àràjòdílálâ fè yèn > [à fórá:!kwâ:ràjòd'lálá: fě:j'] il ne l'a pas trouvé chez le réparateur de radio

à fórá à kó ègilìzî lá > [à fórá:!kóèg 'lìzí:!lá] il ne l’a pas trouvé à l'église

\section{LES CONJONCTIONS EN $11^{\mathrm{ĖRE}}$ POSITION}

\subsection{LA CONJONCTION wálà EN $1{ }^{\mathrm{E} R E}$ POSITION}

4.1.1. La conjonction wálà en $1^{\text {ère }}$ position - pronoms en $2^{\text {ème }}$ position:

Lorsque la conjonction wálà est en première position et les pronoms en deuxième position, l'assimilation a lieu partout sans exception:

wálà í bí táá? > [wálì:b'tá:] ou bien tu pars?

wálà ò bí táá? > [wálò:b'tá:] ou bien celui-là part?

\subsubsection{La conjonction wálà en $1^{\text {ère }}$ position - noms propres en $2^{\text {ème }}$ position:}

Lorsque la conjonction wálà est en première position et les noms propres en deuxième position, l'assimilation peut avoir lieu seulement quand la voyelle initiale du nom propre est $i$ : 
wálà ísá bí táá, ... > [wálì:sáb'tá:] [wálàísáb'tá:] ou bien Issa part, ...

wálà úmù bí nà, ... > [wálàúm bí nà] ou bien Oumou vient, ..

\subsubsection{La conjonction wálà en $1^{\text {ère }}$ position - mots étrangers en $2^{\text {ème }}$ position:}

Lorsque la conjonction wálà est en première position et les mots étrangers en deuxième position, l'assimilation n'a lieu nulle part:

wálà è rè yôn má yé? > [wálà è rèjố:!má jé] ou bien on n'a pas retrouvé le rayon (de bicyclette)?

wálà j̀ròbû tí kálá bílèn? > [wálà j̀̀̀ेb'tí kálá b'lè] ou bien on ne coud plus la robe?

\subsection{LA CONJONCTION wáásá EN 1 ĖRE POSITION}

4.2.1. La conjonction wáásá en $1^{\text {ére }}$ position - pronoms en $2^{\text {ème }}$ position:

Quand la conjonction wáásá est en première position et les pronoms en deuxième position, on constate que l'assimilation a lieu partout sans exception:

..., wáásá ê ká táá > [wá:sé:!ká tá:] ..., afin que toi tu partes

..., wáásá ù ká táá > [wá:sû: ká tá:] ..., afin qu'ils partent

4.2.2. La conjonction wáásá en $1^{\text {ére }}$ position - noms propres en $2^{\text {ème }}$ position:

Quand la conjonction wáásá est en première position et les noms propres en deuxième position, on peut constater que l'assimilation n'est pas observée:

..., wáásá ísá ká táá > [wá:sá ísá ká tá:] ..., afin qu’Issa parte

..., wáásá úmù ká nà $>$ [wá:sá úm ká nà] ..., afin qu'Oumou vienne

4.2.3. La conjonction wáásá en $1^{\text {ére }}$ position - mots étrangers en $2^{\text {ème }}$ position:

Quand la conjonction wáásá est en première position et les mots étrangers en deuxième position, force est de constater que l'assimilation n'a lieu nulle part:

..., wáásá è rè yôn kànà kárí > [wá:sá èrèjjé:!kànà kár'] ..., afin que le rayon ne soit cassé

..., wáásá òròbû ká kálá > [wá:sá j̀ròb'!ká kálá] ..., afin que la robe soit cousue

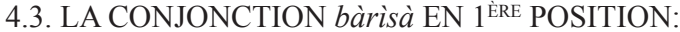

\subsubsection{La conjonction bárìsà en $1^{\text {ère }}$ position - pronoms en $2^{\text {ème }}$ position}

Quand la conjonction bàrì̀à est en première position et les pronoms en deuxième position, on constate que l'assimilation a généralement lieu:

..., bàrìsà ò má táá > [bàr’sô: má tá:] [bàr`sáò má tá:] ..., parce que celui-là n'est pas parti

..., bàrìsà ù má wúlí $>$ [bàr’sû: má wúlí] [bàr’sáù má wúlí] ..., parce qu'ils ne se sont pas levés

\subsubsection{La conjonction bàrìsà en $1^{\text {ère }}$ position - noms propres en $2^{\text {ème }}$ position}

Quand la conjonction bàrì̀à est en première position et les noms propres en deuxième position, on peut constater que l'assimilation n'a lieu nulle part:

..., bàrìsà ísá má wúlí > [bàr`sà ísá má wúlí] ..., parce que Issa ne s'est pas levé

..., bàrìsà úmù má wúlí > [bàr`sà úm má wúlí] ..., parce qu'Oumou ne s'est pas levée 
4.3.3. La conjonction bàrìsà en $1^{\text {ère }}$ position - mots étrangers en $2^{\text {ème }}$ position

Quand la conjonction bàrìsà est en première position et les mots étrangers en deuxième position, force est de constater que l'assimilation n'a lieu nulle part:

..., bàrìsà ègìlìzî má jò > [bàr’sá èg 'lìzí:!má jò] ..., parce que l'église n’a pas été construite

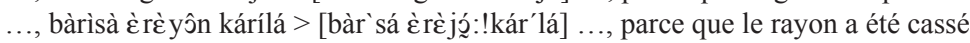

\section{LES ADVERBES EN $1{ }^{\text {ÈRE }}$ POSITION}

\subsection{L'ADVERBE yàn EN $1{ }^{\mathrm{E} R E}$ POSITION}

5.1.1. L'adverbe yàn en $1^{\text {ère }}$ position - pronoms en $2^{\text {ème }}$ position:

Quand l'adverbe yàn est en première position et les pronoms en deuxième position, force est de constater que toute possibilité d'assimilation est exclue quel que soit le pronom en jeu. A noter que l'adverbe yàn perd sa nasalité:

à bí bìlà yàn ê kóró > [àb'b 'lá jàé:!kóró] on le laisse ici à côté de toi

à bí bìlà yàn í kóró > [àb'b lá jàì kóró] on le laisse ici à côté de toi

5.1.2. L'adverbe yàn en $1^{\text {ère }}$ position - noms propres en $2^{\text {ème }}$ position:

Quand l'adverbe yàn est en première position et les noms propres en deuxième position, on fait le même constat que sous 5.1.1 ci-dessus:

à bí bìlà yàn ísá kóró > [àb'b 'lá jàisá kóró] on le laisse ici à côté d'Issa

à bí bìlà yàn úmù kóró > [àb'b lá jàúm kóró] on le laisse ici à côté d'Oumou

\subsubsection{L'adverbe yàn en $1^{\text {ère }}$ position - mots étrangers en $2^{\text {ème }}$ position:}

Quand l'adverbe yàn est en première position et les mots étrangers en deuxième position, on fait le même constat que sous 5.1.1 et 5.1.2 ci-dessus:

à bí bìlà yàn ègìlizî báárákélâ kóró > [àb'b lá jàèg lìzí:!bá:rákélá:!kóró] on le laisse ici à côté de l’ouvrier de l'église

à bí bìlà yàn ìzínî kónó > [àb'b 'lá jàizíń!kónó] on le laisse ici dans l'usine

\subsection{L'ADVERBE yèn EN $1{ }^{\text {ĖRE }}$ POSITION}

5.2.1. L'adverbe yèn en $1^{\text {ère }}$ position - pronoms en $2^{\text {ème }}$ position:

Quand l'adverbe yèn est en première position et les pronoms en deuxième position, on constate que l'assimilation n'a nulle part lieu. A noter aussi que yèn perd sa nasalité:

à bí à fèèrè yèn à yé > [à bá: fè̀:ré jèà jé] il le vend là-bas pour lui

à bí à fèèrè yèn ù yé > [à bá: fè̀:ré jèù jé] il le vend là-bas pour eux

5.2.2. L'adverbe yèn en $1^{\text {ère }}$ position - noms propres en $2^{\text {ème }}$ position:

Quand l'adverbe yèn est en première position et les noms propres en deuxième position, on fait le même constat que sous 5.2.1 ci-dessus: 
à bí à fèèrè yèn àlî yé > [à bá: fè:ré jèàlî: jé] il le vend là-bas pour Ali

à bí à fèèrè yèn úmù yé > [à bá: fè:ré jèúm jé] il le vend là-bas pour Oumou

\subsubsection{L'adverbe yèn en $1^{\text {ère }}$ position - mots étrangers en $2^{\text {ème }}$ position:}

Quand l'adverbe yèn est en première position et les mots étrangers en deuxième position, on fait le même constat que sous 5.2.1 et 5.2.2 ci-dessus:

à bí à fèèrè yèn àràjòdílálâ yé > [à bá: fè:ré jèàràłòd'lálá:!jé] il le vend là-bas pour le réparateur de radio à bí à fèèrè yèn ìzínî báárákélâ yé > [à bá: fè:ré jè̀zín!bá:rákélá:!jé] il le vend là-bas pour l'ouvrier de l'usine

\subsection{L'ADVERBE tàn EN 1 ĖRE POSITION}

\subsubsection{L'adverbe tàn en $1^{\text {ère }}$ position - pronoms en $2^{\text {ème }}$ position:}

Quand l'adverbe tàn est en première position et les pronoms en deuxième position, on peut constater que l'assimilation a lieu en général. A noter que l'adverbe tàn perd sa nasalité:

à tí sé kà ké tàn ê jê lá > [àt'sé kà ké tě:!nć:!ná] [àt'sé kà ké tàé:!nć:!ná] on ne peut pas le faire comme ça devant toi

à tí sé kà ké tàn ù nê lá > [àt'sé kà ké tù: né:!ná] [àt'sé kà ké tàù né:!ná] on ne peut pas le faire comme ça devant eux

\subsubsection{L'adverbe tàn en $1^{\text {ìre }}$ position - noms propres en $2^{\text {ème }}$ position:}

Quand l'adverbe tàn est en première position et les noms propres en deuxième position, force est de constater que l'assimilation n'est pas observée:

à tí sé kà ké tàn ísá nê lá > [àt'sé kà ké tàísá né:!ná] on ne peut pas le faire comme ça devant Issa à tí sé kà ké tàn úmù nê lá > [àt' sé kà ké tàúm né:!ná] on ne peut pas le faire comme ça devant Oumou

\subsubsection{L'adverbe tàn en $1^{\text {ère }}$ position - mots étrangers en $2^{\text {ème }}$ position:}

Quand l'adverbe tàn est en première position et les mots étrangers en deuxième position, on peut faire le même constat déjà fait sous 5.3.2 ci-dessus:

à tí sé kà ké tàn ègilìzî báárákélâ nê lá > [àt'sé kà ké tàèg 'lìzí:!bá:rákélá:!nć:!ná] on ne peut pas le faire comme ça devant l'ouvrier de l'église

à tí sé kà ké tàn ìzínî báárákélâ nê lá > [àt'sé kà ké tàizín!bá:rákélá:!né:!ná] on ne peut pas le faire comme ça devant l'ouvrier de l'usine

\subsection{L'ADVERBE tèn EN 1 ĖRE POSITION}

\subsubsection{L'adverbe tèn en $1^{\text {ère }}$ position - pronoms en $2^{\text {ème }}$ position:}

Quand l'adverbe tèn est en première position et les pronoms en deuxième position, on peut observer que l'assimilation a généralement lieu. A noter que l'adverbe tèn aussi perd sa nasalité:

à tí sé kà ké tèn à jê lá > [àt'sé kà ké tà: né:!ná] [àt'sé kà ké tèà né:!ná] on ne peut pas le faire de cette façon-là devant lui 
à tí sé kà ké tèn ò nê lá > [àt'sé kà ké tò: né:!ná] [àt'sé kà ké tèò né:!ná] on ne peut pas le faire de cette façon-là devant celui-là

\subsubsection{L'adverbe tèn en $1^{\text {ère }}$ position - noms propres en $2^{\text {ème }}$ position:}

Quand l'adverbe tèn est en première position et les noms propres en deuxième position, force est de constater que l'assimilation n'est observée en général que lorsque la voyelle initiale du nom propre est $a$ :

à tí sé kà ké tèn àlî nê lá > [àt'sé kà ké ta:lî: né:!ná] [àt'sé kà ké tèàlî: né:!ná] on ne peut pas le faire de cette façon-là devant Ali

à tí sé kà ké tèn ísá nê lá > [àt'sé kà ké tèisá né:!ná] on ne peut pas le faire de cette façon-là devant Issa

\subsubsection{L'adverbe tèn en $1^{\text {ère }}$ position - mots étrangers en $2^{\text {ème }}$ position:}

Quand l'adverbe tèn est en première position et les mots étrangers en deuxième position, on peut faire le même constat déjà fait sous 5.4.2 ci-dessus:

à tí sé kà ké tèn àràjòdílálâ nê lá > [àt'sé kà ké ta:ràjòd'lálá:!né:!ná] [àt'sé kà ké tèàràjòd'lálá:!nć:!ná] on ne peut pas le faire de cette façon-là devant le réparateur de radio

à tí sé kà ké tèn ìzínî báárákélâ nê lá > [àt'sé kà ké tèizín!bá:rákélá:!né:!ná] on ne peut pas le faire de cette façon-là devant l'ouvrier de l'usine

\subsection{LE NOMINAL só FAISANT FONCTION D’ADVERBE EN $1{ }^{\text {ĖRE }}$ POSITION}

5.5.1. Le nominal só faisant fonction d'adverbe en $1^{\text {ère }}$ position - pronoms en $2^{\text {ème }}$ position:

Quand le nominal só faisant fonction d'adverbe est en première position et les pronoms en deuxième position, on constate la chose suivante:

- Lorsque le pronom est $a$ ou $e$, l'assimilation a lieu avec la présence nécessaire de l'approximant $w$. Par contre, quand le pronom est $i$ ou $u$, l'assimilation n'aura pas lieu:

à táárá só à kámà > [à tá:rá swá:!kámà] il est allé l'attaquer à la maison

à táárá só ù kámà > [à tá:rá sóù kámà] il est allé les attaquer à la maison

5.5.2. Le nominal só faisant fonction d'adverbe en $1^{\text {ère }}$ position - noms propres en $2^{\text {ème }}$ position:

Quand le nominal só faisant fonction d'adverbe est en première position et les noms propres en deuxième position, on constate que l'assimilation a lieu seulement si la voyelle initiale du nom propre est $a$. Dans ce cas, la présence de l'approximant $w$ sera obligatoire:

à táárá só àlî kámà > [à tá:rá swâ:lî: kámà] il est allé attaquer Ali à la maison

à tááá só úmù kámà > [à tá:rá sóúm kámà] il est allé attaquer Oumou à la maison

5.5.3. Le nominal só faisant fonction d'adverbe en $1^{\text {ère }}$ position - mots étrangers en $2^{\text {ème }}$ position:

Quand le nominal só faisant fonction d'adverbe est en première position et les mots étrangers en deuxième position, on constatera que l'assimilation n'a lieu que lorsque la 
voyelle initiale du mot étranger est $a$. Là aussi, l'approximant $w$ sera obligatoirement présent:

à táárá só àràjòdílálâ kámà > [à tá:rá swâ:ràjòd'lálá:!kámà] il est allé attaquer le réparateur de radio à la maison

à táárá só ègìlizî báárákélâ kámà > [à tá:rá sóèg lìzí:!bá:rákélá:!kámà] il est allé attaquer l’ouvrier de l’église à la maison

\section{LA PARTICULE jàá EN $1^{\text {ÈRE }}$ POSITION}

\subsection{LA PARTICULE jàá EN $1^{\text {ĖRE }}$ POSITION - PRONOMS EN $2^{\text {ĖME }}$ POSITION:}

Quand la particule jàá est en première position et les pronoms en deuxième position, on constate que l'assimilation a généralement lieu:

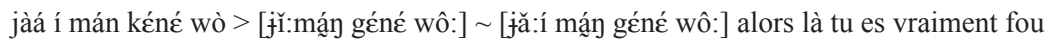
jàá ù má sé gálé $>$ [ju:má sé gálé] [jă:ù má sé gálé] ils ne sont pas encore arrivés à ce que je vois

\subsection{LA PARTICULE jàá EN $1^{\text {ÈRE }}$ POSITION - NOMS PROPRES EN 2 $2^{\text {ÈM }}$ POSITION:}

Quand la particule jàá est en première position et les noms propres en deuxième position, on peut faire le constat suivant: Aucune voyelle initiale des noms propres ne s'assimile à elle:

jàa ísá má bó > [jă:ísá má bó] Issa n'est pas sorti à ce que je vois

jàa úmù má bó > [łă:úm má bó] Oumou n'est pas sortie à ce que je vois

\subsection{LA PARTICULE $j a ̀ a ́$ EN $1^{\text {ĖRE }}$ POSITION - MOTS ÉTRANGERS EN $2^{\text {ĖME }}$ POSITION:}

Quand la particule jàá est en première position et les mots étrangers en deuxième position, force est de constater que l'assimilation n'a lieu nulle part:

jàá èrè gílî sànnà > [jă:èrè g'lí: sànnà] la règle a été achetée à ce que je vois jàá ìzínî fèèrèlà $>$ [jă:ìíń fè:rèlà] l'usine a été vendue à ce que je vois

\section{LE CAS DU MORPHÈME DU PLURIEL $u$}

Le morphème du pluriel est représenté par un $u$ phonologique dont le ton dépend, à notre avis, du mot au quel il est suffixé. Nous nous permettrons de rappeler en passant que dans le parler décrit ici le morphème du pluriel peut être suffixé non seulement à un nom déterminé mais aussi à un nom indéterminé. Par conséquent il ne sera donné ici que des exemples de nominaux en forme indéterminée.

$$
\begin{aligned}
\text { jélé }+\mathrm{u} & >\text { [jéléú] des haches } \\
\text { npálán }+\mathrm{u} & >\text { [mbáláúv }] \text { des sacs } \\
\text { jàbìbì }+\mathrm{u} & >\text { [jàbìbìù }] \text { des ananas } \\
\text { màlì }+\mathrm{u} & >\text { [màlù: }] \text { des hippopotames } \\
\text { dílí }+\mathrm{u} & >\text { [d'líu }] \text { des racines } \\
\text { fìnì }+\mathrm{u} & >\text { [fìnù: }] \text { des habits }
\end{aligned}
$$




$$
\begin{aligned}
& \text { ní }+\mathrm{u}>\text { níú] des âmes } \\
& \text { térí }+\mathrm{u}>\text { [térú:] des amis } \\
& \text { shí }+\mathrm{u} \quad>\text { [Jíú] des poils } \\
& \text { mìshì }+\mathrm{u}>\text { [mìjù:] des bovins }
\end{aligned}
$$

A l'exception de la voyelle finale $i$, aucune autre voyelle finale orale ou nasalisée d'un nominal, qu'il soit déterminé ou indéterminé, ne s'assimile au morphème du pluriel $u$.

Donc, en d'autres termes, on peut parler d'assimilation seulement si la voyelle finale du nominal est $i$. Mais puisque l'assimilation ne semble pas être observée dans tous les cas de nominal à voyelle finale $i$, on tentera de savoir quand l'assimilation a lieu entre le morphème du pluriel $u$ et la voyelle finale $i$ d'un nominal.

Pour qu'il y ait assimilation, il faut qu'une condition bien précise soit remplie. Cette condition est de toute évidence la qualité de la consonne qui précède la voyelle finale $i \mathrm{du}$ nominal. L'enquête, pour autant que nous pouvions la faire, nous a permis d'exclure toutes les autres consonnes et de ne retenir que les consonnes suivantes: $l, n, r$, et $s h$. Pour $l, n$ et $s h$ les restrictions suivantes doivent être faites: dans le cas de $l$, si dans un nominal la voyelle $i$ qui le précède tombe, l'assimilation devient impossible; pour ce qui concerne $n$ et $s h$, l'assimilation ne sera pas possible du tout si le nominal est un monosyllabe (cf. les exemples ci-dessus).

\section{LE CAS DU MORPHÈME DISTRIBUTIF $o ́$}

Il s'agit ici du morphème distributif entre deux nominaux identiques (toujours de forme indéterminée) qui est représenté en général en bamanankan par la séquence vocalique $o$ de ton haut. Nous ajouterons que cette représentation est purement orthographique. Sa structure doit être toute autre. Notre intention n'est pas de discuter en définitif sur le statut phonologique de ce morphème, mais plutôt de décrire si possible son comportement vis à vis du phénomène de l'assimilation. Considérons les exemples suivants:

$$
\begin{aligned}
& \text { bálá ó bálá > [bálá wó:!bálá] chaque balafon } \\
& \text { kònò ó kònò > [kònò wó: kònò] chaque oiseau } \\
& \text { wárí ó wárí > [wáró:!wár'] [wár'wó:!wár'] tout argent } \\
& \text { fàlì ó fàlì } \quad>\text { [fàló: fàl'] [fàl’wó: fàl'] chaque âne }
\end{aligned}
$$

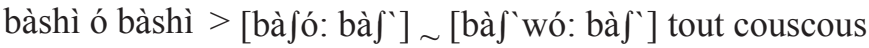

$$
\begin{aligned}
& \text { súkú ó súkú > [súkó:!súkú] [s'kú wó:!súkú] n’importe quel marché } \\
& \text { wùlù ó wùlù }>\text { [wùló: wùlù] } \sim \text { [w`lù wó: wùlù] chaque chien } \\
& \text { túlú ó túlú }>\text { [t'lú wó:! t'lú] toute huile }
\end{aligned}
$$

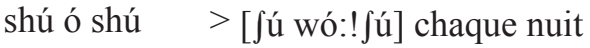

Quant à la question de savoir si l'assimilation a lieu entre la voyelle finale d'un nominal et le morphème distributif, on est tenté d'affirmer au premier abord que le morphème distributif s'assimile en général aux voyelles finales $i$ et $u$ et que l'assimilation n'a jamais lieu quand il s'agit des autres voyelles finales des nominaux ou si le nominal à voyelle finale $i$ et $u$ est un monosyllabe ou se réalise comme tel. 
Nous voulons affirmer ici qu'il n'y a pas d'assimilation entre aucune voyelle finale d'un nominal et le morphème distributif, pour la raison suivante: Si nous regardons les réalisations phonétiques, on constate que: 1) le morphème distributif a une consonne initiale $w$ et une longue voyelle partout où l'assimilation ne semble pas être observée; 2) le downstep est provoqué chez les nominaux de ton haut; 3 ) les nominaux dissyllabiques de ton bas ont leur dernière syllabe relevée au contact du morphème distributif. C'est en fait ce troisième constat qui est la principale raison qui nous fait faire l'affirmation ci-dessus, car un ton bas n'est jamais relevé devant un ton haut. En fait la voyelle finale du nominal est tombée et a laissé la place au morphème distributif dont la voyelle longue a le ton haut.

Le downstep nous dit qu'il doit y avoir quelque part dans le morphème distributif un ton bas (qui deviendrait ensuite flottant).

Cette argumentation nous conduit à poser alors comme hypothèse ceci: le morphème distributif serait une séquence de structure cvv dont les voyelles auraient un ton haut suivi par un ton bas flottant dont le comportement est identique à celui du ton bas flottant de l'élément de la détermination. On se rend bien compte que le morphème distributif a une consonne initiale, si on ralentit le débit du langage avec surtout un nominal à voyelle finale $o$. Donc, tout en attendant de prouver la validité de cette hypothèse, le morphème distributif devrait être représenté de la façon suivante: wóó .

\section{D’AUTRES CAS D’ASSIMILATION}

\section{Ouverture de voyelles}

Les exemples ci-dessous nous fournissent encore un cas d'assimilation où l'on parlerait d'ouverture de voyelle. Les deux premiers montrent que la voyelle finale du premier mot consécutif ou de la première composante du composé, só, s'assimile à la voyelle du deuxième mot consécutif ou de la deuxième composante du composé. Dans le dernier exemple, on assiste d'abord à une assimilation entre la voyelle finale $i$ de $n i$ et le pronom $o$. Ensuite ce produit s'assimile à la voyelle finale $\varepsilon$ de $t \varepsilon ́$ pour devenir une voyelle ouverte:

$$
\begin{aligned}
& \text { sô kó fè > [só:!kó fè] derrière la maison } \\
& \text { maison-De derrière postp. } \\
& \text { só }+ \text { kó }+ \text { fè }+ \text { la }>\text { [sókófélá] derrière la maison } \\
& \text { maison }+ \text { derrière }+ \text { postp. }+ \text { suffixe }
\end{aligned}
$$

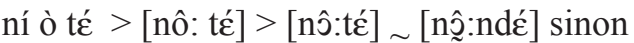

$$
\begin{aligned}
& \text { si celui-là n'est pas }
\end{aligned}
$$

Arrondissement de voyelles

Les exemples ci-dessous sont encore une preuve pour ce cas d'assimilation où l'on parlerait d'arrondissement de voyelle. Ce genre d'assimilation se produit non seulement dans un mot simple mais aussi dans un composé:

$$
\begin{aligned}
& \text { màkò }>[\text { mòkò }] \sim[\text { màkò }] \text { besoin } \\
& \text { nìn }+ \text { lù }>[\text { nùnụ̂: }] \text { ceux-ci } \\
& \text { celui-ci }+ \text { morphème du pluriel }
\end{aligned}
$$


Fermeture de voyelles

Un cas d'assimilation où l'on pourrait parler de fermeture de voyelle nous est fourni ici par l'exemple ci-dessous. Il y'a là un problème d'interprétation quant à la nature du morphème qui provoque la fermeture de la voyelle du premier mot. BAILLEUL (2000:10) l'a répertorié comme «un suffixe hors série» sous la forme - $n i$ et il pense que c'est une «pure marque lexicale qui n'ajoute rien au sens de la base». Nous ne sommes pas de cet avis. Mais ce problème là ne peut pas être discuté ici:

$$
\begin{aligned}
& \text { jón }+ \text { nìn }>\text { [jóǹ }] \sim[\text { jónì }] \text { qui? } \\
& \text { qui }+ \text { ceci? }
\end{aligned}
$$

Cas de produit d'assimilation

Dans l'exemple ci-dessous, nous avons la forme emphatique de la deuxième personne du singulier qui semble être le produit d'assimilation du composé ílè. La forme ile comme forme emphatique de la deuxième personne du singulier se retrouve par exemple dans le parler de Kankan, de Faranah et dans celui de Kuranko (BIRD 1982). Le processus de 1'assimilation est le suivant: La consonne $l$ de ilè tombe de sorte que les deux voyelles qui étaient précédemment séparées par lui se succèdent favorisant ainsi l'assimilation vocalique régressive:

$$
\begin{aligned}
& \hat{\mathrm{e}}<\mathrm{i}+\text { lè } \\
& \text { toi toi }+ \text { particule d'emphase }
\end{aligned}
$$

\section{BIBLIOGRAPHIE}

BAilleul Charles. 1981. Petit Dictionnaire bambara-français, français-bambara. London: Avebury Publishing Co.

Bailleul Charles. 2000. Cours pratique de bambara. Sons - Types de phrases - Tons. Bamako: Editions Donniya.

BIRD Charles S. (ed.). 1982. The Dialects of Mandekan. African Studies Program, Indiana University.

BRAUnER Siegmund. 1974. Lehrbuch des Bambara. Leipzig: Verlag Enzyklopädie.

CReISSEls Denis. 1985. «Les verbes statifs dans les parlers manding». Mandenkan 10, Automne, 1-32.

Diallo Mohamed Larabi. 1989. Zur Verbalstruktur und Syntax des Bambara. Dissertation, Bayreuth.

Diallo Mohamed Larabi. 2003. «L'assimilation en bamanankan». Mandenkan 38, Printemps, 15-45.

DiAllo Mohamed Larabi. 2004a. «L'assimilation vocalique régressive en bamanankan». Mandenkan, 39, Printemps, 23-46.

Diallo Mohamed Larabi. 2004b (2007). «L'assimilation vocalique régressive en bamanankan: d'autres cas». Annali 64, Università Degli Studi Napoli «L'Orientale», Napoli, 1-27.

DoumBIA Salabary. 1987. Untersuchung zur phonologischen Struktur zweier Bambara-Varianten (Bambara von Bamako und Wasolonkan). Dissertation, Bayreuth.

Dumestre Gérard. 1994. Le bambara du Mali: Essai de description linguistique. T. 1 et 2. (Les documents de Linguistique Africaine). Paris: Association Linguistique Africaine.

Greenberg Joseph H. 1963. «The Languages of Africa». Part II. International Journal of American Linguistics 29(1).

Sauvant Émile. 1956. Grammaire Bambara. Ancienne grammaire de Mgr. Sauvant refondue et complétée par Mgr. Molin. Les Presses missionnaires. Issy-Les-Moulineaux. 